\title{
Laringitis fúngica en pacientes inmunocompetentes
}

\author{
Fungal laryngitis in immunocompetent patients
}

\author{
Tomás Andrade $\mathbf{D}^{1}$, Diego Correa $\mathrm{F}^{2}$, Norma León $\mathbf{M}^{3}$, Carla Napolitano $\mathbf{V}^{1}$, Pedro Badía $\mathbf{V}^{1}$.
}

\section{RESUMEN}

Introducción: La laringitis fúngica es una patología poco planteada en pacientes inmunocompetentes, sin embargo se debería tener en consideración en el diagnóstico diferencial de leucoplaquias en estos pacientes, más aún con factores predisponentes como reflujo faringolaríngeo, tabaquismo crónico y/o uso de corticoides.

objetivo: Presentar una serie de casos de pacientes inmunocompetentes con diagnóstico clínico de laringitis fúngica y tratamiento antimicótico empírico.

Describir la asociación con factores predisponentes claves.

Material y método: Estudio retrospectivo que incluyó a 11 pacientes con diagnóstico clínico de laringitis fúngica por correlación de la clínica, factores predisponentes y hallazgos en la videoestroboscopía laríngea (leucoplaquias múltiples en los pliegues vocales) sumado a la respuesta a tratamiento empírico con fluconazol oral. Se realizó además una revisión de la literatura disponible hasta el año 2015.

Resultados: Todos los diagnósticos fueron clínicos correlacionando síntomas con hallazgo de leucoplaquias características en la laringe. El principal factor asociado fue el reflujo faringolaríngeo (91\%) seguido por uso de corticoides (55\%). Todos los pacientes fueron tratados con un esquema empírico de fluconazol oral por 14-21 días. El 100\% de los pacientes respondió de forma exitosa al uso de este fármaco con remisión de los síntomas y de las lesiones laríngeas.

Conclusión: El diagnóstico clínico y tratamiento con fluconazol oral como tratamiento de primera línea generarían buena tasa de respuesta, siempre que se correlacionen los síntomas y signos del paciente con los hallazgos encontrados en la laringe.

Palabras clave: Laringitis, inmunocompetencia, fúngico, corticoesteroides, reflujo faringolaríngeo, tratamiento antifúngico empírico, leucoplaquia, cuerdas vocales, pliegues vocales.

\section{ABSTRACT}

Introduction: The fungal laryngitis is an unusual disease in immunocompetent patients, however should take into consideration in the differential diagnosis of leukopla-

\footnotetext{
Médico Departamento de Otorrinolaringología, Pontificia Universidad Católica de Chile.

2 Médico-Cirujano. Facultad de Medicina. Pontificia Universidad Católica de Chile.

3 Fonoaudióloga. Pontificia Universidad Católica de Chile.
}

Recibido el 4 de septiembre de 2015. Aceptado el 21 de diciembre de 2015. 
kias, especially in patients with predisposing factors such as pharyngolaryngeal reflux, use of inhaled, oral or intravenous corticosteroids.

Aim: Describe a series of cases of fungal laryngitis in immunocompetent patients with clinical diagnosis and empirical antifungal treatment. In addition, finding the association with predisposing factors keys.

Material and method: Retrospective study of 11 patients with diagnosis of fungal laryngitis according to clinical presentation, predisposing factors and findings in the laryngeal videostroboscopy (vocal folds leukoplakias) joined the response to empirical treatment with oral fluconazole. Also an extensive literature review was conducted until 2015.

Results: The main predisposing factor was the pharyngolaryngeal reflux (91\%) followed by use of corticosteroids (55\%).

All patients were treated empirically with fluconazole for 14-21 days.

$100 \%$ of patients responded successfully, with remission of symptoms and laryngeal lesions.

Conclusion: Clinical diagnosis and treatment with fluconazole as first-line treatment generate good response rate, provided that the patient's symptoms and signs with the findings in the larynx are correlated.

Key words: Laryngitis, Fungal, Immunocompetence, steroid, pharyngolaryngeal reflux, empirical antifungal treatment, vocal folds leukoplakias.

\section{INTRODUCCIÓN}

La laringitis fúngica es una patología frecuente en pacientes inmunosuprimidos, puede imitar enfermedades granulomatosas, reflujo faringolaríngeo (RFL), lesiones premalignas, incluso cáncer laríngeo y forma parte importante del diagnóstico diferencial de las leucoplaquias laríngeas ${ }^{1}$. Rara vez se plantea en pacientes inmunocompetentes generando diagnósticos tardíos y tratamientos inadecuados.

La etiología más común de laringitis fúngica es la infección por Cándida albicans, existiendo reporte de casos de Cryptococcus, Histoplasma, Blastomyces y Aspergillus ${ }^{1-9}$.

Dentro de los factores predisponentes tenemos aquellos que deterioran la barrera mucosa local de la laringe como el reflujo faringolaríngeo (RFL), el tabaco, los corticoides inhalados, la radioterapia y el trauma (intubación), además existen los que alteran la respuesta inmune, tales como la inmunosupresión, medicamentos inmunosupresores y déficit nutricionales ${ }^{10-12}$.

En relación a las manifestaciones clínicas de esta patología, existe una correlación entre los tres tipos de signos visibles a la nasofibroscopía con los síntomas presentes en el paciente, así frente a leucoplaquias difusas con mucosa eritematosa subyacente se presenta disfonía; ante un edema y eritema en hipofaringe pseudomembranas blancas o grisáceas se presenta dolor y disfagia; y frente a edema y eritema epiglótico se observa disnea ${ }^{11,13}$.

El diagnóstico puede ser microbiológico 0 clínico. En el primero de los casos sería a través de una biopsia y/o cultivo de la lesión, métodos sólo disponibles en pocos pacientes dado su alto costo, además traería riesgos de injuria cordal y anestésicos, por lo que sería justificable en casos refractarios a tratamiento o sospecha de malignidad. En el caso del diagnóstico clínico se debe realizar correlación entre los síntomas, factores predisponentes, hallazgos en la videonasofibroscopía y respuesta positiva a tratamiento empírico con antimicóticos orales. Dado los menores costos y la ausencia de riesgos del diagnóstico clínico es que esta última manera ha ido ganando gran fuerza en los últimos años ${ }^{14-17}$.

\section{OBJETIVOS}

Describir una serie de casos con diagnóstico clínico de laringitis fúngica en pacientes inmunocompetentes evaluados en la Unidad de Voz de la Red de Salud UC-Christus.

Identificar la asociación con factores predisponentes en esta patología. 


\section{MATERIAL Y MÉTODO}

Serie retrospectiva de casos evaluados y tratados en la Unidad de Voz de nuestro departamento entre octubre de 2012 y junio de 2014 con diagnóstico clínico por hallazgos de leucoplaquias múltiples en los pliegues vocales en la videoestroboscopía laríngea y con respuesta favorable a tratamiento empírico de laringitis fúngica en pacientes inmunocompetentes. El seguimiento clínico fue realizado con estroboscopía laríngea en seis pacientes y con nasofibroscopía en cinco pacientes, y se realizó hasta octubre de 2014.

El tratamiento consistió en el uso de fluconazol vía oral, $300 \mathrm{mg}$ el primer día, luego $150 \mathrm{mg}$ al día por 14 a 21 días. Se realizó control con nasofibroscopía a los 14 días, si existía respuesta completa se suspendía fluconazol, pero si existía respuesta parcial se extendía el tratamiento por 7 días más. Además se realizó estudio previo y posterior al uso del fármaco con pruebas hepáticas.

Las variables se describen a través de promedios o frecuencias porcentuales.

Se rescataron datos epidemiológicos como edad y sexo, manifestaciones clínicas, factores predisponentes y respuesta al tratamiento empírico.

\section{RESULTADOS}

Once pacientes fueron diagnosticados de laringitis fúngica, con edad promedio de 62,7 años (23-87 años). De ellos, el $55 \%$ de sexo femenino y el $45 \%$ masculino.

Todos los diagnósticos fueron clínicos, correlacionando la clínica con el hallazgo de leucoplaquias características en la laringe previamente descritas.

El principal motivo de consulta fue disfonía en $82 \%$ de los pacientes (9 pacientes), $9 \%$ de los pacientes consultó por disfagia y otro $9 \%$ de pacientes por síntomas faringolaríngeos de reflujo gastroesofágico (globus faríngeo, carraspera, odinofagia matinal). Además se observó un tiempo promedio de 6 meses entre la aparición de los síntomas y el diagnóstico e inicio de tratamiento.

Dentro de los factores predisponentes, el reflujo faringolaríngeo (RFL) fue el más frecuente, apareciendo en 10 pacientes (91\%), de estos 9 , tenían diagnóstico previo de RFL y estaban siendo tratados con distintos inhibidores de la bomba de protones: $55,5 \%$ con esomeprazol $40 \mathrm{mg}$ cada 12 horas, 33,5\% con omeprazol $20 \mathrm{mg}$ cada 12 horas y $11 \%$ con lansoprazol $30 \mathrm{mg}$ cada 12 horas.

El paciente en que se diagnosticó RFL en forma concomitante a la laringitis fúngica se le indicó tratamiento con esomeprazol $40 \mathrm{mg}$ cada 12 horas (Figura 1).

Otro factor predisponente prevalente fue el uso de corticoides en $55 \%$ de los pacientes. De ellos, dos pacientes usaban corticoides inhalados (budesonida $200 \mathrm{mcg}, 2$ puff cada 12 horas); 3 pacientes corticoides inhalados y sistémicos (2 con fluticasona 250 mcg 2 puff cada 12 horas + prednisona 5 y $10 \mathrm{mg}$ al día respectivamente y 1

Tabla 1. Resumen de casos con laringitis fúngica

\begin{tabular}{|rccccc|}
\hline Caso & Edad & Sexo & Síntomas & Factores predisponentes & Ubicación anatómica \\
\hline 1 & 60 & M & Disfonía & RFL & Glotis \\
2 & 55 & M & Disfonía & RFL + INH + SIST & Glotis \\
3 & 87 & M & Disnea & RFL + SIST & Supraglotis + Glotis \\
4 & 71 & F & Disfonía & INH + SIST & Glotis \\
5 & 44 & F & Disfonía + odinofagia & RFL + SIST & Glotis \\
6 & 23 & M & Disfagia & RFL & Supraglotis + Glotis \\
7 & 81 & F & Disfonía & RFL + DM & Glotis \\
8 & 59 & M & Disfonía & RFL & Glotis \\
9 & 61 & F & Disfonía & RFL + INH + SIST & Supraglotis + Glotis \\
10 & 85 & F & Disfonía + odinofagia & RFL + INH & Glotis \\
11 & 64 & F & Disfonía &
\end{tabular}

(INH: Corticoides inhalados; SIST: Corticoides sistémicos; RFL: Reflujo faringolaríngeo; DM: Diabetes Mellitus). 


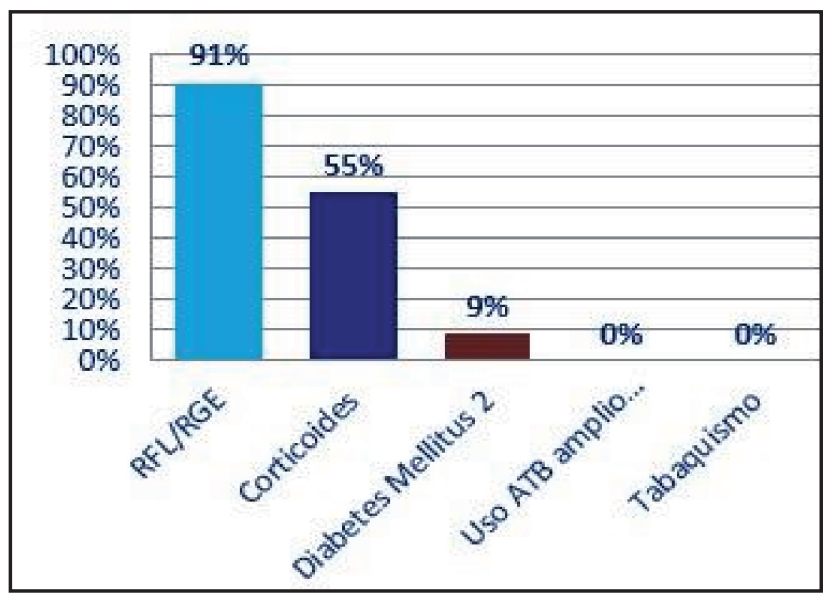

Figura 1. Factores predisponentes en pacientes con laringitis fúngica.

con budesonida $200 \mathrm{mcg}, 2$ puff cada 12 horas + prednisona $7.5 \mathrm{mg}$ al día); y 1 paciente sólo con corticoides sistémicos (prednisona 7,5 mg al día) (Figura 2).

También destacó la diabetes mellitus tipo 2 presente en $9 \%$ de los pacientes.

Ningún paciente fumaba ni había utilizado antibióticos previos.

En relación a otros antecedentes mórbidos, el $50 \%$ de los pacientes tenía alguna enfermedad pulmonar crónica (2 asma, 2 EPOC y uno fibrosis pulmonar por exposición laboral), todos ellos en tratamiento; $30 \%$ de los pacientes tenía hipotiroidismo.

En cuanto al compromiso anatómico, en $73 \%$ de los pacientes había solo compromiso de la glotis, de ellos la mitad tenía lesiones en ambas cuerdas vocales. El $27 \%$ restante de los casos presentaba leucoplaquias en la supraglotis y glotis en forma sincrónica. No se observó compromiso subglótico (Figura 3).

Todos los pacientes fueron tratados con un esquema empírico de fluconazol vía oral, $300 \mathrm{mg}$ el primer día, luego $150 \mathrm{mg}$ al día por un lapso de 14 a 21 días (7 pacientes por 14 días y 4 por 21 días). A todos los pacientes se les realizó estudio de pruebas hepáticas al inicio del tratamiento y posterior a éste, resultando todos normales.

El $100 \%$ de los pacientes respondió en forma exitosa al uso de fluconazol oral, con remisión de los síntomas y de las lesiones larín-

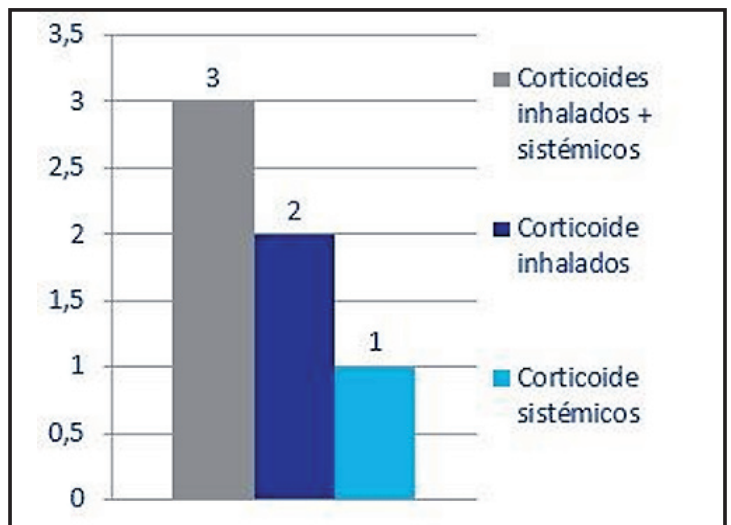

Figura 2. Uso de corticoides en pacientes con laringitis fúngica.



Figura 3. Compromiso anatómico en pacientes con laringitis fúngica. 
geas (Figura 4). Sólo un paciente persistió con leucoplaquias en glotis a las 3 semanas de tratamiento por lo que se solicitó biopsia y cultivo. Al realizar la microlaringoscopía de suspensión diagnóstica, 2 semanas después de su indicación, no había evidencia de lesiones laríngeas y la biopsia del epitelio de la cara superior del pliegue vocal (inicialmente comprometido) resultó sin displasia ni elementos micóticos en la biopsia. Por esto se consideró como una respuesta tardía al tratamiento antifúngico.

Ningún paciente ha reportado alguna recidiva luego de 12 meses de seguimiento promedio.

\section{DISCUSIÓN}

Treinta y tres casos similares han sido reportados desde 1968 hasta el 2015 (Tabla 2) con edad promedio 56,7 años (33-77 años). El 77\% de los casos fue en sexo femenino. El motivo de consulta más frecuente fue la disfonía similar a nuestra experiencia ${ }^{1,3-5,9,10-13,15-32}$.
En relación a los factores predisponentes, hubo cierta diferencia, ya que en nuestro estudio el más frecuente fue el reflujo faringolaríngeo a diferencia de los corticoides inhalados que fueron el factor que más destacó en la revisión de la literatura, incluso se describe que $10 \%$ a $15 \%$ de los pacientes con corticoides inhalados reportan laringitis fúngica y que la incidencia de disfonía por ellos llegaría hasta el 55\%. Ambos factores tienen en común la forma en que facilitan la invasión por hongos: la destrucción de la mucosa local10,11,18-20.

Por otra parte, en nuestra muestra, cuatro pacientes refieren el uso de corticoides sistémicos, lo que se podría considerar como pacientes inmunosuprimidos, sin embargo todos usaban dosis bajo el equivalente de inmunosupresión (prednisona $0,15 \mathrm{mg} / \mathrm{kg} / \mathrm{al}$ día por más de 3 meses).

Al analizar el compromiso anatómico, la mayoría de los estudios muestran casos con lesiones en glotis y subglotis, pero además aparecen tres casos de compromiso de epiglotis que se manifes-

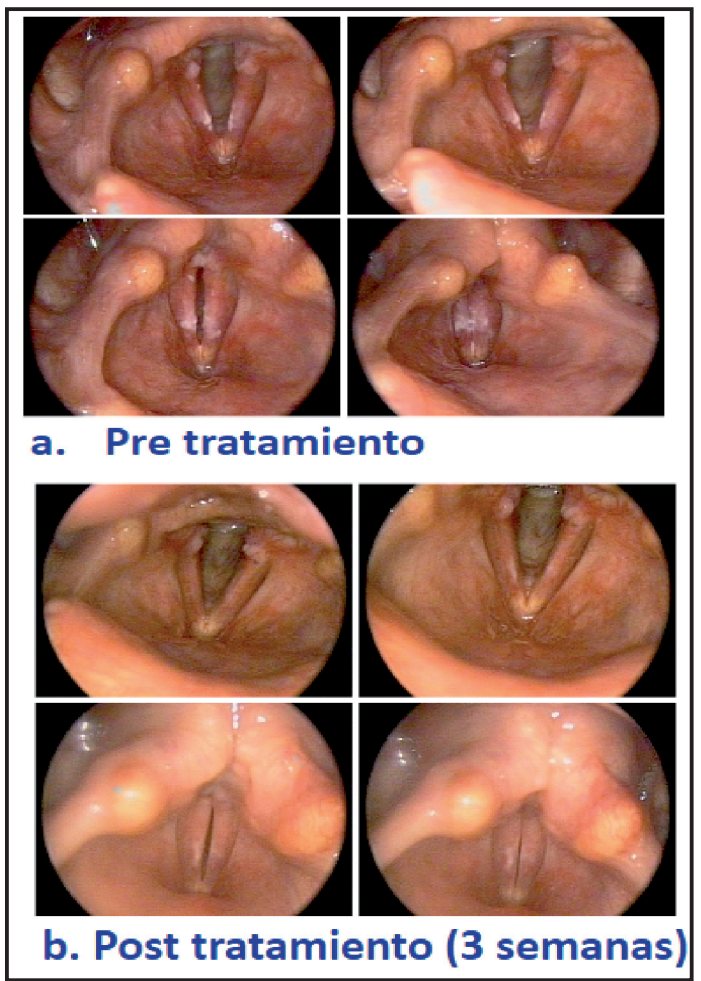

Figura 4. Respuesta a tratamiento empírico con fluconazol en pacientes con laringitis fúngica. 
Tabla 2. Reportes de casos de laringitis fúngica publicados entre el año 1968 al 2015

\begin{tabular}{|c|c|c|c|c|c|c|c|}
\hline Autores & Edad & Sexo & Síntomas & $\begin{array}{c}\text { Factores } \\
\text { predisponentes }\end{array}$ & $\begin{array}{l}\text { Ubicación } \\
\text { anatómica }\end{array}$ & Diagnóstico & Tto \\
\hline Tedeschi and Cheren ${ }^{22}$ & 42 & M & Disfonía & TBQ & Glotis & Biopsia & $\mathrm{N}+\mathrm{G}$ \\
\hline Yonkers ${ }^{23}$ & 65 & $\begin{array}{c}\mathrm{M} \\
+ \text { disfagia }\end{array}$ & Disfonía + Dolor & $\begin{array}{c}\text { ATB } \\
+ \text { Subglotis }\end{array}$ & Supraglotis+Glotis & Biopsia & N \\
\hline Hicks and Peters ${ }^{15}$ & 56 & $\mathrm{~F}$ & Disfonía & $N / E$ & Glotis & Biopsia & G \\
\hline Haberman et al ${ }^{21}$ & 75 & $\begin{array}{c}F \\
+ \text { disnea }\end{array}$ & Dolor+ disfagia & $\mathrm{N} / \mathrm{E}$ & Epiglotis & - & - \\
\hline Selkin ${ }^{9}$ & 64 & $\begin{array}{c}F \\
+ \text { disnea }\end{array}$ & Dolor+ disfagia & $\begin{array}{c}\text { N/E } \\
+ \text { Subglotis }\end{array}$ & Supraglotis+Glotis & Biopsia & K \\
\hline Forrest and Weed ${ }^{1}$ & 38 & $\mathrm{~F}$ & Disfonía & $\mathrm{N} / \mathrm{E}$ & Glotis & Biopsia & $\mathrm{F}$ \\
\hline Fairfax et a $\left.\right|^{28}$ & 75 & M & Disfonía & INH + ATB & Glotis & Biopsia & A \\
\hline Zhang et a $\left.\right|^{29}$ & 60 & M & Disfonía & $\begin{array}{c}\text { SIST } \\
+ \text { +Subglotis }\end{array}$ & Supraglotis+Glotis & Biopsia & $\mathrm{F}$ \\
\hline Neuen-Schwander et al ${ }^{16}$ & 33 & $\mathrm{~F}$ & Disfonía + disfagia & SIST & Supraglotis + Glotis & Clínico & $\mathrm{F}$ \\
\hline Del Gaudio ${ }^{12}$ & 41 & $\mathrm{~F}$ & Disfonía & $\begin{array}{c}\text { INH } \\
\text { +Subglotis }\end{array}$ & Supraglotis+Glotis & Biopsia & $\mathrm{F}$ \\
\hline De Pasquale and Sataloff ${ }^{30}$ & 50 & $\mathrm{~F}$ & Disfonía + disfagia & $\begin{array}{l}\text { SIST +ATB } \\
\text { +Subglotis }\end{array}$ & Supraglotis+Glotis & Biopsia & $\mathrm{F}$ \\
\hline Scheid et a $\left.\right|^{32}$ & 52 & $\mathrm{~F}$ & Disfonía & $\mathrm{TBQ}$ & Glotis (úlcera) & Cultivo & $\mathrm{F}$ \\
\hline Mehanna, Hisham M., et al ${ }^{10}$ & 52 & M & Disfonía & $\mathrm{TBQ}+\mathrm{RFL}$ & Glotis & Biopsia & $\mathrm{F}$ \\
\hline Mehanna, Hisham M., et al ${ }^{10}$ & 44 & $\mathrm{~F}$ & Disfonía & $T B Q+R F L+I N H$ & Glotis & Biopsia & N \\
\hline Mehanna, Hisham M., et al ${ }^{10}$ & 73 & $\mathrm{~F}$ & Disfonía & $\mathrm{TBQ}+\mathrm{INH}$ & Glotis & Biopsia & K \\
\hline Sulica et a $\left.\right|^{13}$ & 71 & $\mathrm{~F}$ & Disfonía & SIST & Supraglotis + Glotis & Biopsia & $\mathrm{F}$ \\
\hline Sulica et a $\left.\right|^{13}$ & 75 & $\mathrm{~F}$ & Disfonía & INH & Glotis & Biopsia & $\mathrm{F}$ \\
\hline Sulica et al ${ }^{13}$ & 53 & $\mathrm{~F}$ & Disfonía + Dolor & $\begin{array}{l}\text { SIST + INH } \\
\text { +Subglotis }\end{array}$ & Supraglotis+Glotis & Clínico & $\mathrm{F}$ \\
\hline Sulica et al ${ }^{13}$ & 62 & M & Disfonía + Dolor & $\begin{array}{l}\mathrm{DM}+\mathrm{ATB} \\
+ \text { Subglotis }\end{array}$ & Supraglotis+Glotis & Clínico & $\mathrm{F}$ \\
\hline Sulica et al ${ }^{13}$ & 59 & $\mathrm{~F}$ & Disfonía & $\mathrm{SIST}+\mathrm{INH}$ & Supraglotis + Glotis & Clínico & $\mathrm{F}$ \\
\hline Sulica et a $\left.\right|^{13}$ & 60 & $\mathrm{~F}$ & Disfonía & INH & Glotis & Clínico & $\mathrm{F}$ \\
\hline Sulica et al13 & 77 & $\mathrm{~F}$ & Disfonía & INH & Glotis & Clínico & $\mathrm{F}$ \\
\hline Nunes, Fabio P., et al. ${ }^{5}$ & 58 & M & $\begin{array}{c}\text { Disfonía + Disnea } \\
+ \text { ATB }\end{array}$ & $\begin{array}{l}\text { TBQ + INH } \\
+ \text { Glotis }\end{array}$ & Supraglotis (E) & Biopsia & $\mathrm{F}$ \\
\hline Hirshoren et al. ${ }^{4}$ & 50 & $\begin{array}{c}\text { M } \\
+ \text { Disfagia }\end{array}$ & Disfonía + Disnea & TBQ & Supraglotis (E) & Cultivo & $\mathrm{F}$ \\
\hline Chandran, Swapna K., et al. ${ }^{27}$ & 65 & $\mathrm{~F}$ & $\begin{array}{c}\text { Disfonía } \\
+ \text { INH }\end{array}$ & $\mathrm{RFL}+\mathrm{TBQ}$ & Supraglotis + Glotis & Clínico & $\mathrm{F}+\mathrm{N}$ \\
\hline Nair, Arun B., et al ${ }^{31}$ & 73 & $\begin{array}{c}M \\
+ \text { fiebre }\end{array}$ & Odinofagia + disnea & $\mathrm{N} / \mathrm{E}$ & Supraglotis + Glotis & Biopsia & $\mathrm{F}$ \\
\hline Malton et al. ${ }^{26}$ & 47 & $\mathrm{~F}$ & $\begin{array}{l}\text { Disnea } \\
+ \text { SIST }\end{array}$ & $\begin{array}{c}\mathrm{DM}+\mathrm{INH} \\
+ \text { +Glotis+Subglotis }\end{array}$ & Supraglotis & Clínico & $\mathrm{F}$ \\
\hline Heman-Ackah et al ${ }^{17}$ & 41 & M & Disfonía + tos & INH + RFL & Glotis & Clínico & $\mathrm{F}+\mathrm{N}$ \\
\hline Lee et al ${ }^{24}$ & 62 & M & Disfonía & TBQ & Glotis + Subglotis & Biopsia & । \\
\hline Ravikumar, A., et al ${ }^{25}$ & 34 & $\mathrm{~F}$ & Disfonía + Tos & $N / E$ & Glotis & Biopsia & I \\
\hline Ravikumar, A., et al 25 & 52 & $\mathrm{~F}$ & $\begin{array}{l}\text { Disfonía + Dolor } \\
\text { + Disfagia + tos }\end{array}$ & $N / E$ & Supraglotis + Glotis & Cultivo & I \\
\hline
\end{tabular}

(N: Nistatina; G: Genciana violeta; F: Fluconazol; A: Anfotericina; I: Itraconazol; K: Ketoconazol) ${ }^{1,3-5,9,10-13,15-32}$ 
taron sólo con disnea, mientras en nuestro estudio no hubo ninguno $0^{4,5,21}$.

En relación al tratamiento, éste ha ido cambiado a lo largo de los años. Inicialmente, se utilizó la nistatina y la violeta genciana, para luego dar paso a los triazoles, como el ketoconazol, itraconazol y fluconazol. Este último, ha sido considerado como tratamiento de primera línea en los últimos 10 años, dada la buena respuesta y que no se ha reportado resistencia $1^{1,3-5,9,10,12,16,17,22-27,29-32}$.

La principal controversia del último tiempo es en relación a la forma de realizar el diagnóstico de la laringitis fúngica. Si bien el diagnóstico definitivo se realiza con biopsia y cultivo, la poca disponibilidad y los riesgos que ésta puede traer han hecho que esta opción se deje para casos refractarios y durante los últimos ocho casos de laringitis fúngica reportados en la literatura entre los años 2001 al 2012 se optó por el diagnóstico clínico más tratamiento antifúngico empírico con excelente respuesta en la remisión de síntomas y lesiones en laringe ${ }^{13,17,26-27}$.

\section{CONCLUSIÓN}

La laringitis fúngica en pacientes inmunocompetentes es una patología poco frecuente por lo que tiende a ser subdiagnosticada, por ello debemos incorporarla dentro de nuestro diagnóstico diferencial de leucoplaquias en pacientes con clínica y factores predisponentes descritos.

Además, la aproximación diagnóstica conservadora parece ser una excelente opción en esta patología, al igual que el uso de fluconazol como tratamiento de primera línea.

Sin embargo, faltan estudios clínicos que comparen la efectividad de ambos tipos de aproximaciones diagnósticas.

\section{BIBLIOGRAFÍA}

1. Forrest LA, and Harrison W. Candida laryngitis appearing as leukoplakia and gerd. Journal of Voice 1998; 12(1): 91-5.

2. Alba D, Perna C, Molina F, Ortega L, Varquez JJ. Isolated laryngeal candidiasis. Description of 2 cases and review of the literature. Archivos de bronconeumologia, 1996; 32(4): 205-8.

3. Mäkitie AA, Bäck L, Aaltonen LM, Leivo I, Valtonen $M$. Fungal infection of the epiglottis simulating a clinical malignancy. Archives of OtolaryngologyHead \& Neck Surgery 2003; 129(1): 124-6.

4. Hirshoren N, Ron E, and Jeffrey MW. Candida epiglottitis: A rare emergent conditionAppearance, treatment and pitfalls. European Journal of Internal Medicine 2008; 19(8): e84-5.

5. Nunes FP, Bishop T, Prasad ML, Madison JM, Kim DY. Laryngeal candidiasis mimicking malignancy. The Laryngoscope 2008; 118(11): 1957-9.

6. Nadrous HF, Lewis JE, RyuJH, Sabri AN.Cryptococcal laryngitis: case report and review of the literature. Annals of Otology, Rhinology \& Laryngology 2004; 113(2): 121-3.

7. Hanson JM, Gershon S, and Samir K. El-Mofty. Laryngeal Blastomycosis: A Commonly Missed Diagnosis Report of Two Cases and Review of the Literature. Annals of Otology, Rhinology \& Laryngology 2000; 109(3): 281-6.

8. Teoh, Jian Woel, H. Faridah, and Yunus MR Mohamad. Laryngeal histoplasmosis: an occupational hazard. Singapore Medical Journal 2013; 54(10): e208-10.

9. Selkin SG. Laryngeal candidiasis and ketoconazole. Otolaryngology--head and neck surgery: official journal of American Academy of Otolaryngology-Head and Neck Surgery 1985; 93(5): 661.

10. Mehanna HM, Kuo T, Chaplin J, Taylor G, \& Morton RP. Fungal laryngitis in immunocompetent patients. The Journal of Laryngology \& Otology 2004; 118(5): 379-81.

11. Mirza, Natasha, Sandra Kasper Schwartz, and Danielle Antin-Ozerkis. Laryngeal findings in users of combination corticosteroid and bronchodilator therapy. The Laryngoscope 2004; 114(9): 1566-9.

12. DelGaudio, John M. Steroid inhaler laryngitis: dysphonia caused by inhaled fluticasone therapy. Archives of Otolaryngology-Head \& Neck Surgery 2002; 128(6): 677-81.

13. Sulica, Lucian. Laryngeal thrush. Annals of Otology, Rhinology \& Laryngology 2005; 114(5): 369-75.

14. Ganesan S, Harar RPS, Dawkins RS, Prior AJ. Invasive laryngeal candidiasis: a cause of stridor 
in the previously irradiated patient. $J$ Laryngol Otol 1998; 112: 575-8.

15. Hicks JN, Peters GE. Pseudocarcinomatous hyperplasia of the larynx due to Candida albicans. Laryngoscope 1982; 92: 644-7.

16. Neuenschwander MC, Cooney A, Spiegel JR, Lyons KM, \& Sataloff RT. Laryngeal candidiasis: Brief Article. Ear, Nose \&Throat J 2001; 80: 138.

17. Heman-Ackah, Yolanda D., Mary J. Hawkshaw, and Karen M. Lyons. Laryngeal Thrush from Asthma Inhalers. Atlas of Laryngoscopy 2012; 1: 94.

18. Williamson IJ, Matusiewicz SP, Brown PH, Greening AP, \& Crompton GK. Frequency of voice problems and cough in patients using pressurized aerosol inhaled steroid preparations. European Respiratory Journal 1995; 8(4): 590-2.

19. Nishimura K, Koyama H, Ikeda A, Tsukino M, Hajiro T, MIshima M, \& IzUMI T. The effect of high-dose inhaled beclomethasone dipropionate in patients with stable COPD. CHEST Journal 199; 115: 317.

20. Lavy JA, Wood G, Rubin JS, \& Harries M. Dysphonia associated with inhaled steroids. Journal of Voice 2000; 14(4): 581-8.

21. Haberman RS, Becker ME, \& Ford CN. Candida epiglottitis. Archives of Otolaryngology 1983; 109(11): 770-1.

22. Tedeschi LG, CheRen RV. Laryngeal hyperkeratosis due to primary monilial infection. Arch Otolaryngol 1968; 87: 82-4.

23. Yonkers AJ. Candidiasis of the larynx. Ann Otol Rhinol Laryngol 1973; 82: 812-5.
24. Lee DH, Hyong HC. Primary candidiasis and squamous cell carcinoma of the larynx: report of a case. Surgery Today 2013; 2: 203-5.

25. Ravikumar A, Kumar SP, Somu L, Sudhir B. Fungal laryngitis in immunocompetent patients. Indian Journal of Otolaryngology and Head \& Neck Surgery 2014; 66(1): 375-8.

26. Malton A, DeFatta Ra, Sataloff RT. Acute candidal pharyngolaryngitis. Ear, Nose, \& Throat Journal 2012; 91(7): E31.

27. Chandran SK, Lyons KM, Divi V, Geyer M, Sataloff RT. Fungal laryngitis. Ear, Nose, \& Throat Journal 2009; 88(8): 1026.

28. Fairfax AJ, David V, Douce G. Laryngeal aspergillosis following high dose inhaled fluticasone therapy for asthma. Thorax 1999; 54(9): 860-1.

29. Zhang S, Farmer TL, Frable MA, Powers CN. Adult herpetic laryngitis with concurrent candidal infection: a case report and literature review. Archives of Otolaryngology-Head \& Neck Surgery 2000; 126(5): 672-4.

30. De Pasquale K, Sataloff RT. Candida of the larynx. Ear Nose Throat J 2003; 82: 419.

31. Nair AB, Chaturvedi J, Venkatasubbareddy MB, Correa M, Rajan N, Sawkar A. A Case of Isolated Laryngeal Candidiasis Mimicking Laryngeal Carcinoma in an Immunocompetent Individual. The Malaysian Journal of Medical Sciences: MJMS 2011; 18(3): 75.

32. Scheid SC, Anderson TD, Sataloff RT. Ulcerative fungal laryngitis. Ear, Nose, \& Throat Journal 2003; 82(3): 168-9.

Dirección: Dr. Pedro Badía V. 